\section{Hypoplasia of Infrarenal Inferior Vena Cava: An Infrequent Cause of Repetitive Deep Vein Thrombosis and its Effects}

\author{
Keywords: Deep vein thrombosis; Inferior vena cava; Anomalies; \\ Congenital; Agenesis; Hypoplasia; Risk factors

\begin{abstract}
Congenital anomalies such as the defects of inferior vena cava (IVC) are not common, however these alterations obtained from a poor embryo development benefit venous stasis which, in consequence, will trigger a deep vein thrombosis (DVT). This diagnosis must be considered for young patients who have no evident risks of spontaneous and bilateral DVT.
\end{abstract} \\ We introduce a clinical case of a 39 year-old male with \\ iliofemoral DVT who presented recuming DVT after the withdrawal of \\ antic oagulation, with no other predisposing factor.
}

\section{Introduction}

Venous thromboembolic disease (VTD) is an important medical situation that primarily consists of deep vein thrombosis (DVT) and pulmonary embolism (PE). It is the third leading cause of death in the Western world, just behind acute coronary syndrome and cerebrovascular accident. Furthermore, this pathology is associated to a significant morbidity, especially due to post-thrombotic syndrome caused by DVT (20-50\% after DVT) [1] and pulmonary hypertension after PE ( $1 \%$ at 6 months and $3.8 \%$ in 2 years) [2]. The incidence of DVT in the general population is 160 cases per 100,000 and for PE is 60 cases per 100,000, which would result in an overall incidence of VTE in 220 cases per 100,000, with a estimated prevalent average of $3 \%[3,4]$. The incidence of DVT is ten times less frequent in 20 to 40 years old adults [5].

Hypoplasia of inferior vena cava (IVC) constitutes a congenital malformation of the venous system that affects its suprarenal portion. This situation has a very low incidence $(0,0005-1 \%)$ in the general population and somewhat higher in young patients with deep venous thrombosis (DVT) and no other predisposing factor (5\%) [6]. It's been estimated a risk increase $(2 \%)$ in patients with other congenital cardiovascular defects. In most cases, it is usually a casual finding in the image testing (69\%), with DVT being the predominant set of symptoms or chronic venous insufficiency (31\%) [7,8]. In recent years, due to the radiological progress within computerized tomography and magnetic resonance, the anomalies of the vena cava have emerged as a new ethological factor that must be considered [9]. The basis of the treatment consists in long-term or indefinite anticoagulation; other authors suggest invasive procedures through venous endovascular interventional strategies when the location of the anomaly has been clearly identified [10].

\section{Journal of}

Hematology \& Thrombosis

\author{
Rojas Gutiérrez Andrés ${ }^{1}$, Rosa Salazar Vladimir ${ }^{2 *}$, \\ Otálora Valderrama Sonia del Pilar , García \\ Méndez María del Mar², Hernández Contreras \\ María Encarnación ${ }^{2}$ and García Pérez Bartolomé ${ }^{2}$ \\ ${ }^{1}$ Medicina Familiar y Comunitaria, Hospital Clínico Universitario \\ Virgen de la Arrixaca, Murcia, Spain \\ ${ }^{2}$ Unidad de Corta Estancia, Hospital Clínico Universitario Virgen de \\ la Arrixaca, Murcia, Spain \\ ${ }^{3}$ Medicina Interna, Hospital Clínico Universitario Virgen de la \\ Arrixaca, Spain

\section{*Address for Correspondence:} \\ Rosa Salazar Vladimir, Unidad de Corta Estancia, Hospital \\ Clínico Universitario Virgen de la Arrixaca, Murcia, Spain, \\ E-mail: Vladi_medico@yahoo.es \\ Submission: 08 January, 2015 \\ Accepted: 03 March, 2015 \\ Published: 09 March, 2015 \\ Reviewed \& Approved by: Dr. Paolo Prandoni, Professor, \\ Department of Medicine, Thromboembolism Unit, University of Padua, \\ Italy
}

We introduce a clinical case of young male with DVT and hypoplasia of infrarenal inferior vena cava.

\section{Clinical Case}

30 year-old male with no predisposing risk factors of venous thromboembolic disease; he has been diagnosed through a doppler ultrasound of a right iliofemoral-popliteal DVT. Consequently, thrombophilia and anti-cardiolipin antibodies analysis were carried out with a negative result. In the same way, a computed tomography and a phlebography identified a hypoplasia of inferior vena cava. After an early suspension of anticoagulation (withdrawn after a month by the patient himself), he presented, after eight years, another DVT in his iliofemoral vein and his left lumbar vein, which were diagnosed through an ultrasound scan and a computerized tomography, showing hypertrophy of the azygos and hemiazygos systems (Figures 1-3). After a physical examination, it was noted a secondary varicose veins in the abdomen constantly increasing when performing valsalva maneuvers (Figure 4). As a treatment, it was decided to perform indefinite anticoagulation with 4-hydroxycoumarins. After 5 years of evolvement, there is no post-thrombotic syndrome in the lower limbs although the abdominal varicose veins and collateral circulation persisted.

\section{Discussion}

IVC consists of 3 segments of different embryological origin: prerenal, renal and postrenal, resulting from the fusion and partial re absorption of 3 pairs of vessels, which are dependent of the posterior cardinal veins in the embryo. All undertaken studies have shown that the most common anomalies found have been hypoplasia of the prerenal and renal segment, followed by the ones of the post renal segment and the presence of inferior double vena cava [6]. From 
Citation: Andrés RG, Vladimir RS, del Pilar OVS, del Mar GMM, María Encarnación HC, et al. Hypoplasia of Infrarenal Inferior Vena Cava: An Infrequent Cause of Repetitive Deep Vein Thrombosis and its Effects. J Hematol Thromb 2015;1(1): 3.

the embryo's point of view, the prerenal segment comprises the hepatic vein and right sub cardinal vein, the renal segment consists of sub cardinal veins and upper right cardinal veins and the post renal segment composed by the upper right cardinal vein [6]. This complex evolutionary process can lead to anatomic anomalies that will intricate the venous drainage and will favour a development of thrombosis caused by venous stasis. $90 \%$ of the suprarenal portion is affected and only $6-10 \%$ of the renal or infrarenal segment is affected.

Congenital malformations of the IVC are present in $0.3-0.5 \%$ of

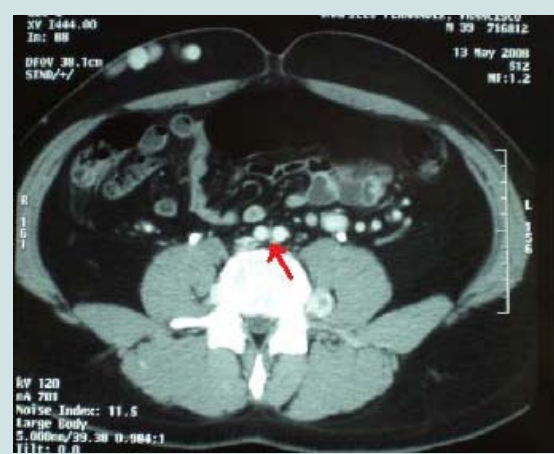

Figure 1: Hypoplasia of Infrarenal inferior vena cava

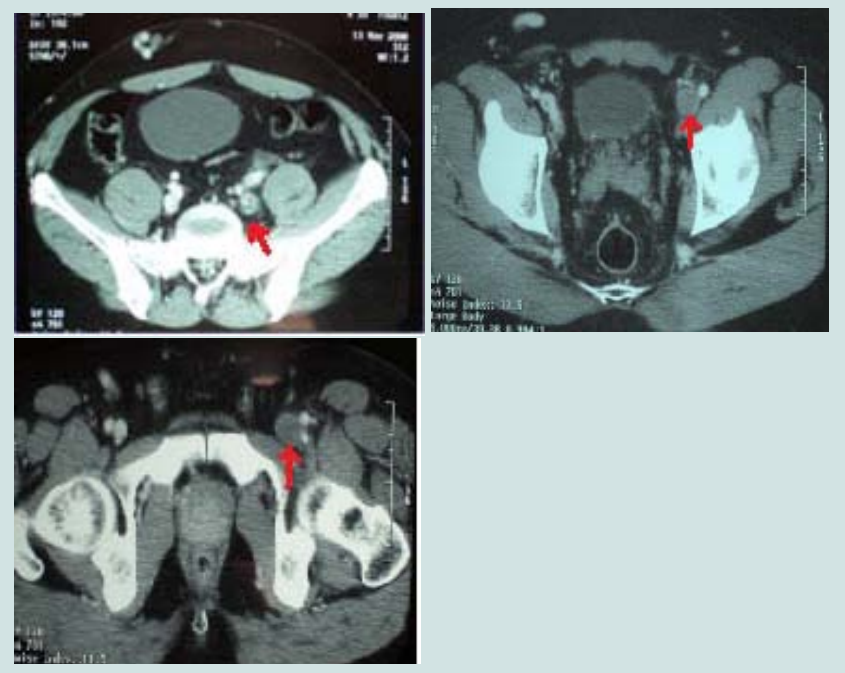

Figure 2: Iliofemoral /left lumbar vein DVT.

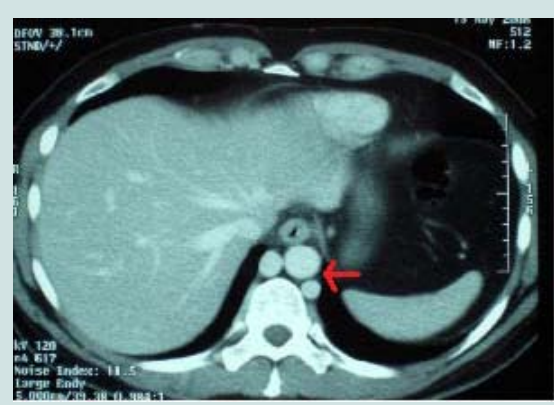

Figure 3: Hypertrophy of the azygos and hemiazygos system.

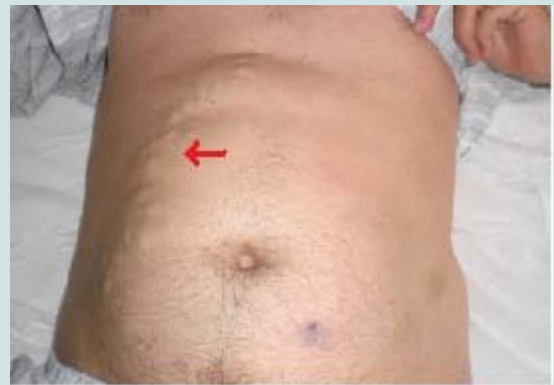

Figure 4: Abdominal varicose vein.

healthy individuals and in $0.6-2 \%$ in patients with other cardiovascular anomalies. The most frequent ones are the presence of a left IVC and the duplication of IVC present in $0.2-3 \%$ of individuals. The agenesis of the IVC has an incidence of $0.0005-1 \%$ in the general population and some recent studies seem to confirm its role as an important factor of predisposition for the development of DVT in young people, with a prevalence of $5 \%$ [11].

Some authors conclude that DVT presented in these areas are caused by interaction between alterations in the coagulation and risk factors obtained due to malformations, surgery, immobilization and pregnancy $[6,9]$. One of the differential diagnosis to be initially discarded is cancer $[12,13]$, which is not the case shown in this discussion, however there are malignant and benign neoplasm that can affect this IVC, consequently triggering DVT.

In young patients with no DVT risk factors, malformations of IVC should be suspected and therefore further analysis must be carried out using image testing. The therapy for lower extremity DVT caused by anomaly of the IVC is typically treated with anticoagulation. There is no data found in publications in reference to the duration of this anticoagulation. Some authors advocate for an indefinite anticoagulation due to the existence of a permanent risk factor of venous stasis caused by malformation $[8,11]$. Some publications propose an invasive endovascular therapy if the location of the anomaly has been clearly identified [8], although the tendency is to maintain anticoagulation indefinitely but further tests are necessary before commencing this treatment.

\section{Conclusions}

After reviewing publications and due to the unusual findings in our patient, for young patients with idiopathic DVT affecting the iliac vein without a clear predisposing factor, it seems reasonable to dismiss IVC malformations with image testing as it could limit the duration of the anticoagulation or contemplate an indefinite treatment, intending to evade the recurrence of a thrombotic event and the morbimortality that it implies.

\section{References}

1. Stain M, Schonauer V, Minar E, Bialonczyk C, Hirschl M, et al. (2005) The post-thrombotic syndrome: risk factors and impact on the course of thrombotic disease. J Thromb Haemost 3: 2671-2676.

2. Piazza G, Goldhaber SZ (2011) Chronic thromboembolic pulmonary hypertension. N Engl J Med 364: 351-360.

3. Anderson FA, Wheeler HB, Goldberg RJ, Hosmer DW, Patwardhan NA, et al. (1991) A Population-based perspective of the hospital incidence and 
Citation: Andrés RG, Vladimir RS, del Pilar OVS, del Mar GMM, María Encarnación HC, et al. Hypoplasia of Infrarenal Inferior Vena Cava: An Infrequent Cause of Repetitive Deep Vein Thrombosis and its Effects. J Hematol Thromb 2015;1(1): 3.

case-fatality rates of deep vein thrombosis and pulmonary embolism. The Worcester DVT Study. Arch Intern Med 151: 933-938.

4. Silverstein MD, Heit JA, Mohr DN, Petterson TM, O’Fallon WM, et al. (1998) Trends in the incidence of deep vein thrombosis and pulmonary embolism. A 25-year population-based study. Arch Intern Med 158: 585-593.

5. Lane DA (2005) Congenital hypoplasia of the inferior vena cava: An underappreciated cause of deep venous thromboses among young adults. Mil Med 170: 739-742.

6. García-Fuster MJ, Forner MJ, Flor-Lorente B, Soler J, Campos S (2006) Anomalías de la vena cava y trombosis venosa profunda. Rev Esp Cardiol 59:171-175.

7. Chaudhary S, Kamyab A, Jacobs M (2013) Congenitally absent inferior vena cava. Am Surg 9: E202-203.

8. Dean SM, Tytle TL (2006) Acute right lower extremity iliofemoral deep venous thrombosis secondary to an anomalous inferior vena cava: A report of two cases. Vasc Med 11: 165-169.
9. Ruggeri M, Tosetto A, Castaman G, Rodeghiero F (2001) Congenital absence of the inferior vena cava: A rare risk factor for idiopathic deep-vein thrombosis. Lancet 357: 441.

10. Atmatzidis K, Papaziogas B, Pavlidis T, Paraskevas G, Mirelis C, et al. (2006) Surgical images: soft tissue. Reccurent deep vein thrombosis caused by hipoplasia of the inferior vena cava. Can J Surg 49: 285.

11. Martínez-Aguilar E, Medina-Maldonado FJ, Flórez GA, Varela-Casariego C, Gómez-Penas M (2008) Hipoplasia de la Vena cava inferior asociada a una trombosis venosa profunda de los miembros inferiores. Angiología 60: 55-59.

12. Zhang L, Yang G, Shen W, Qi J (2007) Spectrum of the inferior vena cava: MDCT findings. Abdom Imaging 32: 495-503.

13. Basile A, Certo A, Ascenti G, Lamberto S, Cannella A, et al. (2003) Embryologic and acquired anomalies of the inferior vena cava with recurrent deep vein thrombosis. Abdom Imaging 28: 400-403. 